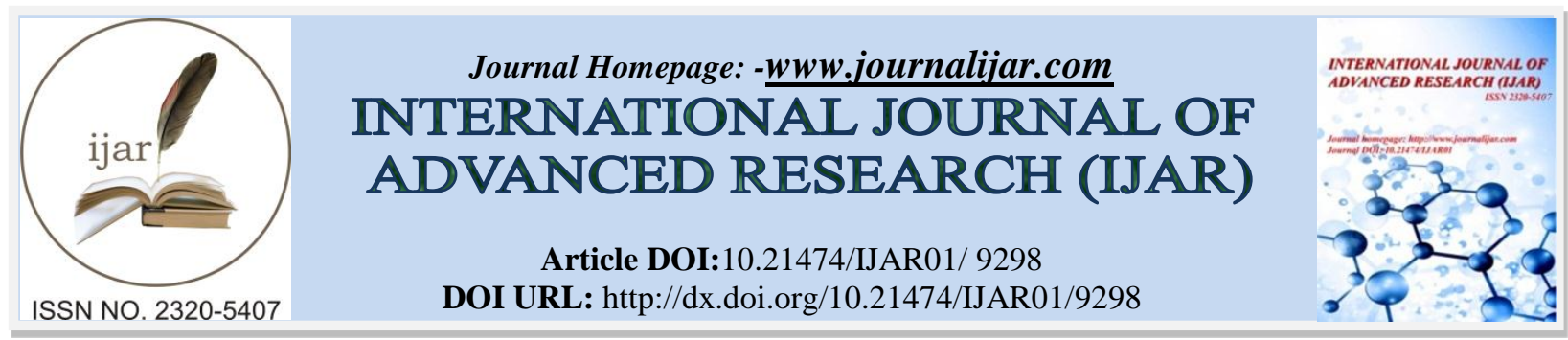

RESEARCH ARTICLE

\title{
EVALUATION OF RADIOMORPHOMETRIC INDICES OF NORMAL AND POSTMENOPAUSAL OSTEOPOROTIC FEMALE PATIENTS BY PANORAMIC RADIOGRAPHS.
}

Ajita Singh, Hina Handa, Bhanupriya Mahant and Christopher Vinay Shinde.

\section{Manuscript Info}

\section{Manuscript History}

Received: 15 April 2019

Final Accepted: 17 May 2019

Published: June 2019

Keywords:-

Bone Mineral Density, Radiomorphometric Indices, Panoramic Mandibular Index (PMI), Mandibular Cortical Index (MCI), and Antegonial index (AI), Bone Mineral Density (BMD).
Abstract

Purpose:To evaluate the significance of radiomorphometric indices, Panoramic Mandibular Index (PMI), Mandibular Cortical Index (MCI) and Antegonial index (AI) of the mandibular bone on panoramic radiographs and co-related with bone mineral density (BMD) for the diagnosis of postmenopausal osteoporosis.

Methedology:Group 'A'- 20 premenopausal women, Age range 30-55 years .Group 'B' - 20 postmenopausal women, Age range 57- 75 years Subjects for the study were selected through detailed case history, clinical examination and BMD scans (DXA machine- WIPRO (GE) LUNAR DPX DXA machine (version 10.5)) has been done for AP lumbar spine (L1-L4), Right and Left femur neck. Analysis of osteoporosis by T-score was done on the basis of WHO classification. Radiographic screening with OPG (Advapex 2000 (Xtropan imaging system version 2000) has been done. Subjects who fulfilled the given clinical and radiographic criteria were subjected to evaluation of radiomorphometric indices, Panoramic Mandibular Index (PMI), Mandibular Cortical Index (MCI), and Antegonial index (AI) through OPG

Results:In our study Bone Mineral Density (BMD/T-scores) in Femur of Post-Menopausal group females $\mathrm{T}$ Score is -1.7 and PreMenopausal female groups $\mathrm{T}$-score is -0.06 . Interobserver agreement has taken for Panoramic mandibular Index, Antegonial Index, Mandibular Cortical Index. PMI is $0.29 \mathrm{~mm}$ and $0.34 \mathrm{~mm}$, AI is $2.96 \mathrm{~mm}$ and $3.58 \mathrm{~mm}$ and $\mathrm{MCI}$ in class $\mathrm{C}_{1}$ is $20 \%$ and $80 \%$ Class $\mathrm{C}_{2}$ is $55 \%$ and $15 \%$ signifies presence of osteopenia Class $\mathrm{C}_{3}$ is $25 \%$ and $5 \%$ signifies presence of osteoporosis for post menopausal and pre menopausal females respectively.

Conclusion:In conclusion, the present study has proved a positive result in predicting osteoporosis by panoramic radiographs. Hence, panoramic radiographs seem to be a simple, inexpensive tool for screening of osteoporosis among the patients coming for dental evaluation.Our results demonstrate that although radiomorphometric indices are regarded as an ancillary method for the diagnosis of osteoporosis on panoramic radiographs 


\section{Introduction:-}

Osteoporosis is one of the most common metabolic bone disorder associated with aging ${ }^{1}$. It is characterized by compromised bone strength, is frequently not detected until a fracture occurs and is considered to be a silent disease that entails significant social and economic burdens ${ }^{2}$. According to World Health Organization (WHO) It is defined as "a skeletal disease, characterized by low bone mass and micro-architectural deterioration of bone tissue leading to enhanced bone fragility, with consequent increase in fracture risk" 1 .

A Gulsahi, et all (2008) Osteoporosis is usually diagnosed by Bone Mineral Density (BMD) measurements, expressed as a T- score. According to WHO criteria, BMD values are divided into following clinical guidelines: normal ( $\mathrm{T}$-score $>-1.0$ ), osteopenia ( $\mathrm{T}$-score between -1.0 and -2.5), and osteoporosis $(\mathrm{T}$-score < -2.5) (5). Osteoporotic fractures are associated with substantial morbidity and high mortality risk involving spine, hip, forearm and proximal humerus and among them hip fractures incur the greatest morbidity and mortality, and give rise to the highest direct costs for health services ${ }^{3}$.

Celia Regina et al (2008) Bone densitometry has been consensually accepted as the golden standard in the diagnosis of osteoporosis ${ }^{4}$. Dual-energy X-ray absorptiometry (DXA) and quantitative ultrasound are among the techniques developed to identify individuals with skeletal osteoporosis. Panoramic radiography has been an important component of dental diagnostic radiology for over 40 years. It is a technique that produces an image of the teeth and jaws on a single film. Nowadays there is a great tendency among researchers to use panoramic radiographs for detection osteoporosis.

F Yasar et al (2009) Panoramic radiography has been an important component of dental diagnostic radiology for over 40 years. It is a technique that produces an image of the teeth and jaws on a single film. Nowadays there is a great tendency among researchers to use panoramic radiographs for detection osteoporosis. Identifying women with low bone mineral density using panoramic radiographs is a particular topic that has drawn the attention of researchers over the last decade.

Leite AF et al (2010) Panoramic radiographic measurements, also known as panoramic radiomorphometric indices, have been developed to assess mandibular cortical shape and width, either qualitatively and quantitatively .Most studies have used Panoramic Mandibular Index (PMI), Antegonial Index (AI) and Mandibular Cortical Index (MCI) , and are significantly associated with BMD of the general skeleton, and risk of osteoporotic fractures in postmenopausal women ${ }^{2}$.

\section{Aim Of The Study}

To evaluate Panoramic Mandibular Index (PMI), Mandibular Cortical Index (MCI), and Antegonial index (AI) of normal and postmenopausal osteoporotic female patients by panoramic radiographs.

\section{Objectives Of The Study}

1. To compare and correlate between measurements of PMI, MCI and AI made from panoramic radiographs and mandibular bone mineral density using DXA.

2. To help in the early detection of osteoporosis by the dental professionals, in order to reduce risk of fracture and high mortality rate.

3. To assess whether panoramic radiographs only be a reliable screening for osteoporosis.

4. To evaluate the most reliable index among PMI, MCI \& AI for diagnosing osteoporosis.

\section{Material And Methods:-}

\section{Armamentarium for clinical examination of patient}

1. Mouth mirror

2. Probe

3. Mask /gloves

4. Autoclave

\section{Armamentarium for radiography}

1. X-ray machine - Advapex 2000 (Xtropan imaging system version 2000) low frequency

2. Printer - Fujifilm capsula plus 4000 dry pix

3. Digital film - Fujifilm $\left(8^{\prime \prime} \times 10^{\prime \prime}\right)$ 
4. Lead apron

5. Lead Barrier

\section{Armamentarium for DXA}

1. DXA machine- WIPRO (GE) LUNAR DPX DXA machine (version 10.51)

2. Scan report

3. Lead apron

\section{Armamentarium for radiographic interpretation}

1. Room with subdued ambient illumination.

2. View box

3. Magnifying lens

4. Vernier Calliper

5. $0.5 \mathrm{~mm}$ Markers

6. Transparency sheets

7. Scale

\section{Methodology:-}

All the individual patients were examined under artificial illumination using mouth mirror and conventional probe. The data regarding menopausal status, age, height, weight and medical history were recorded.

The selected group 'A' and 'B' were subjected for DXA assessment of the hip region (right and left femoral neck) and Lumbar spine (L1 - L4) will be done and Panoramic radiography.

\section{Subjects}

The subjects for this clinical study consist of -

1. Group 'A'-20 premenopausal women (healthy individuals belonging to 30-55 years)

2. Group 'B'- 20 post menopausal women (belonging to age group 57-75 years)

\section{Exclusion Criteria $^{5}$}

1. Pregnancy

2. Hyperthyroidism

3. Hyperparathyroidism

4. Diabetes Mellitus

5. Paget disease

6. Oral bisphosphonates

7. Systemic glucocorticoids

All the individual patients were examined under artificial illumination using mouth mirror and conventional probe. The data regarding menopausal status, age , height, weight and medical history were recorded.

The selected group 'A' and 'B' were subjected for DXA assessment and Panoramic radiography.

\section{Bone Mineral Density Assessment}

All radio-opaque objects should be removed from the scan area (jewelry, belts, etc.).

For whole body scan the subject was positioned in the Centre of the table, aligned with the long axis of the scanner, with their head near the head end of the table. The subject's head positioned straight up, not turned to the left or right. If required for subject comfort, only radiolucent pillows should be used.

The legs and feet were positioned together with a Velcro strap around the ankles to help avoid movement.

Hands were positioned with palms flat against the scan table. Space was maintained between the arms and the torso when possible. Hands should not be tucked under the hips to keep them in the scan field. If necessary, the subject's hands should be taped to the scan table. The knees should not be bent to keep the feet within the scan field.

Subjects were classified according to WHO criteria for osteoporosis depending on T-score defined as follows : 
BMD scans has been done for AP lumbar spine (L1-L4), Right and Left femur neck. Analysis of osteoporosis by Tscore was done on the basis of WHO classification ${ }^{6}$.

The World Health Organization (WHO) criteria for defining osteoporosis, osteopenia and normal is as follows :

1. Normal - BMD for young healthy adult $(\mathrm{T} \geq-1.0)$

2. Osteopenia - BMD for osteopenia ( T score between -1.0 and -2.5 )

3. Osteoporosis $-\mathrm{BMD} \leq 2.5 \mathrm{SD}$

\section{Clinical Protocol}

\section{Before scanning patient}

Before patients come in for their scans, they should be informed of the following issues:

1. It was ensured that patients can tolerate lying flat on their back and keeping still for at least $10 \mathrm{~min}$.

2. The weight of subjects was determined. All DXA systems can scan patients up to $300 \mathrm{lb}(136 \mathrm{~kg})$. If they are over $300 \mathrm{lb}$, they may need to have an alternative bone density or body composition test performed.

3. To check any medical imaging procedure recently if they have received contrast, such as barium or gadolinium, they should be scheduled two weeks after contrast was administered.

4. In premenopausal patients, it was asked that is there any possibility that they might be pregnant.

5. Calcium tablets should not be taken in the $24 \mathrm{~h}$ before the examination.

6. Patients must wear comfortable, loose fitting clothes, such as a sweat suit

\section{Radiographic Procedure}

All Group 'A' and Group 'B' were drapped with lead apron and thyroid collar.

Panoramic radiographs were taken for all patients who gave informed consent at time of DXA measurement with panoramic X-ray machine (Advapex 2000 the Xtropan imaging system version 2000). In order to achieve a standard, the radiographs were obtained by a single examiner at $3-6 \mathrm{~mA}$ and $18 \mathrm{sec}$ the voltage varied between 55 $80 \mathrm{kVp}$ depending on the age, sex and built of the patient. Patients were positioned in the dental panoramic machine in such a way that vertical line produced by the machine was aligned with the patient's saggital plane, with the horizontal line (Frankfort plane) parallel to the floor.

After the exposure, all the images were recorded with the help of a printer (Fujifilm capsula plus 4000 dry pix). Later all the radiographs were submitted for measurement of radiomorphometric indices by two observers independently.

\section{Assessment of Radiographic interpretation}

First each observer traced the structures of interest on transparencies placed on the radiographs. Measurements were bilaterally performed with the help of vernier caliper and scale by using magnifying lens on the transparencies by the same observer.

A second observer performed the same measurement at different time, without any knowledge about the first observer's readings.

All measurements were made with both the observers blinded to the reference osteoporotic diagnosis.

All the measurements were aimed at obtaining the following radiomorphometric indices :

1. Panoramic Mandibular Index (PMI)

2. Antegonial index (AI)

3. Mandibular Cortical Index (MCI),

Panoramic Mandibular Index (PMI) -

The PMI is the ratio of the thickness of the mandibular cortex to the distance between the mental foramen and the inferior mandibular cortex bilaterally ${ }^{7}$. (Fig-1)

\section{Mandibular Cortical Index (MCI) -}

Appearance of the inferior mandibular cortical thickness . MCI was assessed according to the criteria defined by Klemetti et al ${ }^{4}$

$\mathrm{C} 1$ - The endosteal margin of the cortex was even and sharp on both sides (Normal) (fig 2)

$\mathrm{C} 2$ - The endosteal margin shows semilunar defects (lacunar resorption) and /or seems 
to form endosteal cortical residues on one or both sides (mild to moderately eroded cortex) (osteopenia) (fig 3) C3 -The cortical layer forms endosteal cortical residues and was clearly porous (severly eroded) (Osteoporosis) (fig 4)

\section{Antegonial Index (AI) -}

Mandibular cortical thickness measured on the line perpendicular to the mandibular cortical at the intersection with the tangent line to the anterior border of the branch (normal value: $\geq 3.2 \mathrm{~mm}$ ) defined by D Ledgerton and $\mathrm{K}$ Horner ${ }^{6}$ (fig 5)

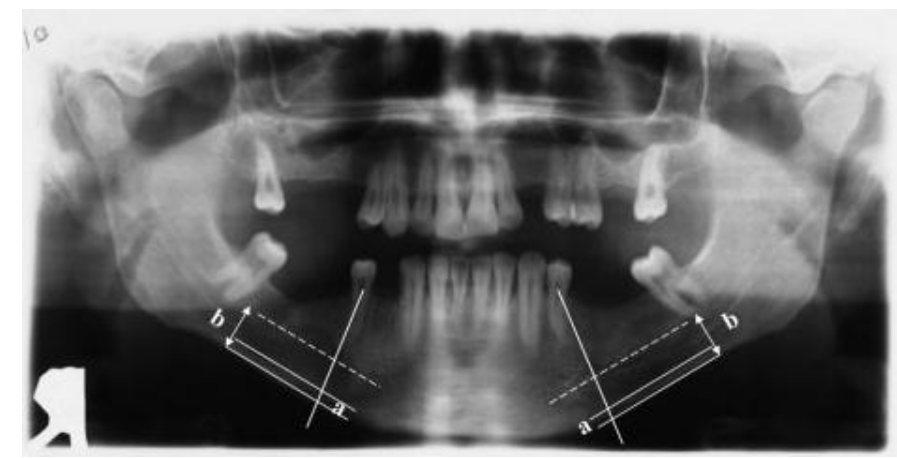

Fig 1:-Panoramic Mandibular Index (PMI)

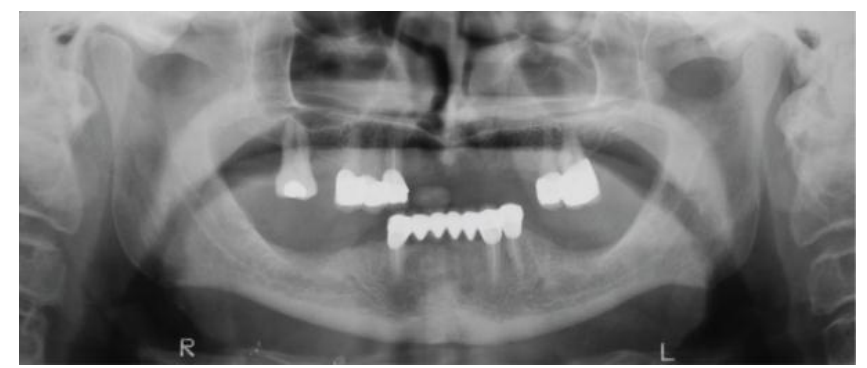

Fig 2:-C1 - The endosteal margin of the cortex was even and sharp on both sides (Normal)

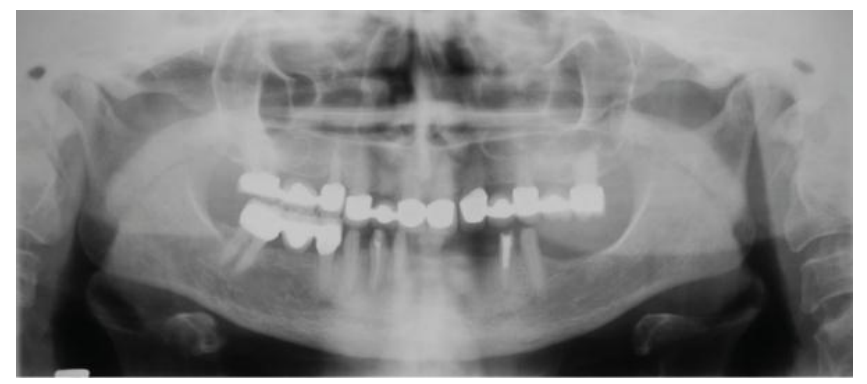

Fig 3:-C2 - The endosteal margin shows semilunar defects (lacunar resorption) and /or seems to form endosteal cortical residues on one or both sides (mild to moderately eroded cortex) (osteopenia)

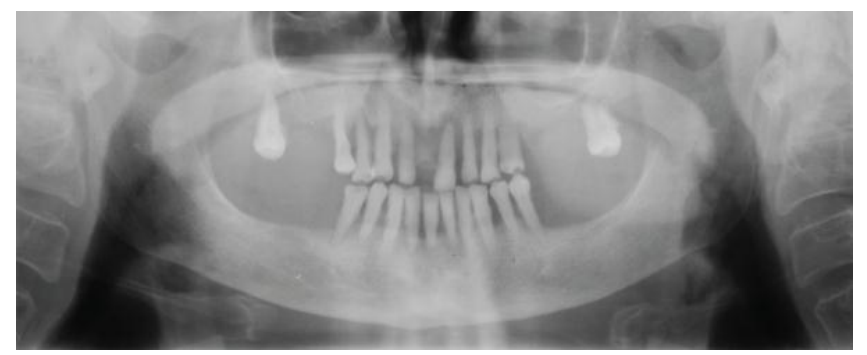

Fig 4:-C3 -The cortical layer forms endosteal cortical residues and was clearly porous (severly eroded) (Osteoporosis) 


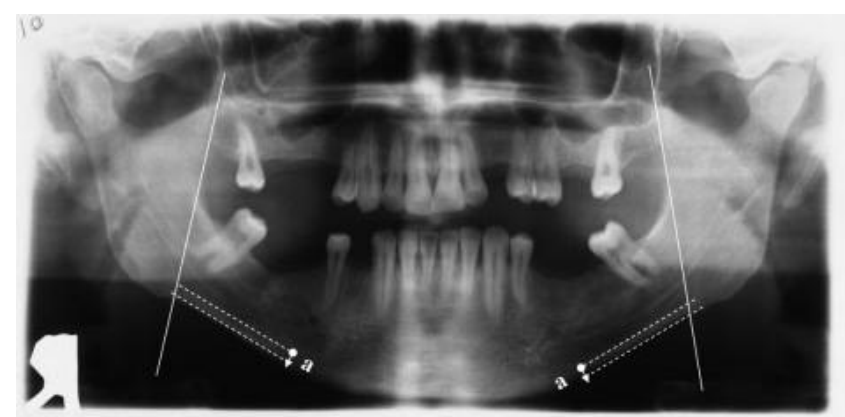

\section{Results:-}

Fig 5:-Antegonial Index (AI)

Age correlation among post menopausal and pre menopausal female patients . The mean Age (in years) was $63.70 \pm$ 8.35 and $45.35 \pm 5.70$ for post-menopausal and premenopausal females respectively $(\mathrm{p}<0.001)$ showing significant difference (Graph 1 ).



Graph 1:-Mean values of Age (in years) in Post-Menopausal and Pre-Menopausal female groups.

Bone Mineral Density /T-score in femur region of post menopausal and pre menopausal female patients.

The mean BMD for femur region was -1.76 and -0.06 for post menopausal and pre menopausal female respectively $(\mathrm{p}<0.001)$ showing that significant difference is present. For post menopausal females Mean T-score of femur stands for osteopenia. The mean BMD for spine region was -3.15 and -0.13 for post menopausal and pre menopausal female patients respectively $(\mathrm{p}<0.001)$ showing the significant difference in Spine T-score.

\section{Interobserver Analysis}

In the present study, Inter-observer agreement for of PMI and AI (continuous data) was evaluated by Pearson correlation coefficient and the correlation between the observers for PMI (post-menopausal: $r=0.917$, preMenopausal: $r=0.712$ ) and AI (post-menopausal: $r=0.936$, pre-Menopausal: $r=0.860$ ) agreements were interpreted as very strong agreement.

Inter-observer agreement for MCI (ordinal data) was evaluated by weighted kappa. The agreement between the observers for MCI (post-menopausal: $\mathrm{k}=1.000$, pre-Menopausal: $\mathrm{K}=1.000$ ) was found to be statistically significant 


\begin{tabular}{|l|l|l|l|}
\hline Agreement for & Test Applied & Test Results & Interpretation \\
\hline $\begin{array}{l}\text { Post-Menopausal: } \\
\text { Panoramic mandibular } \\
\text { Index }\end{array}$ & Pearson Correlation & $\begin{array}{l}\mathrm{r}=0.917 \\
\mathrm{p}=0.000(\mathrm{p}<0.001)\end{array}$ & Very strong Agreement \\
\hline $\begin{array}{l}\text { Post-Menopausal: } \\
\text { Antegonial Index }\end{array}$ & Pearson Correlation & $\begin{array}{l}\mathrm{r}=0.936 \\
\mathrm{p}=0.000(\mathrm{p}<0.001)\end{array}$ & Very strong Agreement \\
\hline $\begin{array}{l}\text { Post-Menopausal: } \\
\text { Mandibular Cortical Index }\end{array}$ & Weighted Kappa & $\mathrm{K}=1.000$ & $\begin{array}{l}\text { Almost } \\
\text { Agreement }\end{array}$ \\
\hline $\begin{array}{l}\text { Pre-Menopausal: Panoramic } \\
\text { mandibular Index }\end{array}$ & Pearson Correlation & $\mathrm{r}=0.000(\mathrm{p}<0.001)$ & Very strong Agreement \\
\hline $\begin{array}{l}\text { Pre-Menopausal: Antegonial } \\
\text { Index }\end{array}$ & Pearson Correlation & $\mathrm{p}=0.000(\mathrm{p}<0.001)$ & Very strong Agreement \\
\hline $\begin{array}{l}\text { Pre-Menopausal: } \\
\text { Mandibular Cortical Index }\end{array}$ & Weighted Kappa & $\mathrm{p}=0.000(\mathrm{p}<0.001)$ & Almost \\
\hline
\end{tabular}

Table 1:-Inter-observer agreement for Panoramic mandibular Index, Antegonial Index, Mandibular Cortical Index.

\section{Panoramic Mandibular Index (PMI)}

Panoramic Mandibular Index (PMI) $<0.30 \mathrm{~mm}$ considered as osteoporotic finding. So, here in this study mean PMI is $0.29 \mathrm{~mm}$ and $0.34 \mathrm{~mm}$ for post menopausal and pre menopausal females respectively and statistically significant difference was observed between the groups $(\mathrm{p}<0.01)$.

\section{Antegonial Index}

Antegonial Index (AI) $<3.2 \mathrm{~mm}$ considered as osteoporotic finding. In the present study mean AI $2.96 \mathrm{~mm}$ and 3.58 for post menopausal and pre menopausal females respectively and statistically significant difference was observed between the groups $(\mathrm{p}<0.001)$

\section{Mandibular Cortical Index (MCI)}

1. Mandibular Cortical Index (MCI) $\mathrm{C}_{1}$ stands for Normal, $\mathrm{C}_{2}$ stand for osteopenia and $\mathrm{C}_{3}$ stands for osteoporosis ${ }^{5}$

2. In the present study $\%$ of MCI in class $\mathrm{C}_{1}$ is $20 \%$ and $80 \%$ for post menopausal and pre menopausal females respectively

3. Class $\mathrm{C}_{2}$ is $55 \%$ and $15 \%$ for post menopausal and pre menopausal females respectively signifies presence of osteopenia

4. Class $\mathrm{C}_{3}$ is $25 \%$ and $5 \%$ for post menopausal and pre menopausal females respectively signifies presence of osteoporosis.

\begin{tabular}{|l|l|l|l|l|l|l|l|l|}
\hline \multirow{2}{*}{ Groups } & Class I & \multicolumn{2}{l|}{ Class II } & Class III & \multicolumn{2}{l|}{ Total } \\
\cline { 2 - 9 } & $\mathbf{n}$ & $\mathbf{6}$ & $\mathbf{n}$ & $\mathbf{\%}$ & $\mathbf{n}$ & $\mathbf{\%}$ & $\mathbf{n}$ & $\mathbf{\%}$ \\
\hline $\begin{array}{l}\text { Post-Menopausal } \\
\text { Female }\end{array}$ & 4 & 20 & 11 & 55 & 5 & 25 & $\mathbf{2 0}$ & $\mathbf{1 0 0}$ \\
\hline $\begin{array}{l}\text { Pre-Menopausal } \\
\text { Female }\end{array}$ & 16 & 80 & 3 & 15 & 1 & 5 & $\mathbf{2 0}$ & $\mathbf{1 0 0}$ \\
\hline Total & $\mathbf{2 0}$ & $\mathbf{5 0}$ & $\mathbf{1 4}$ & $\mathbf{3 5}$ & $\mathbf{6}$ & $\mathbf{1 5}$ & $\mathbf{4 0}$ & $\mathbf{1 0 0}$ \\
\hline Pearson Chi-Square test Value $=14.438, \mathrm{P}=0.001(<0.01)$, Significant Difference \\
\hline
\end{tabular}

Table 2:-Mandibular Cortical Index (MCI) scores for Post-Menopausal and Pre-Menopausal female groups.

\section{Roc Curve}

Receiver Operating Characteristic (ROC) Curve for identifying osteoporosis by Panoramic Mandibular Index. 


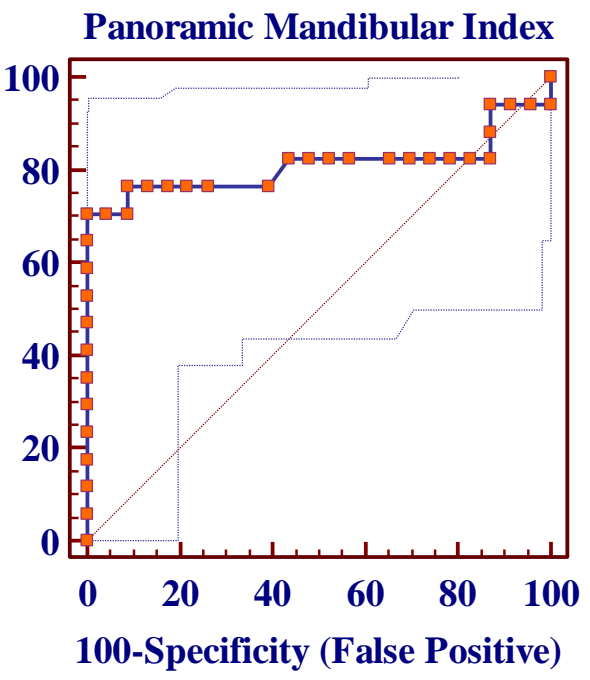

Area under the ROC curve (AUC)

\begin{tabular}{|l|l|}
\hline Area under the ROC curve (AUC) & 0.809 \\
\hline 95\% Confidence interval & 0.654 to 0.916 \\
\hline P value & $0.000(<0.001)$ Significant result \\
\hline
\end{tabular}

Threshold value with highest validity

\begin{tabular}{|l|l|}
\hline Threshold value with Highest validity & $\leq 0.292 \mathrm{~mm}$ \\
\hline Sensitivity & $70.59(95 \% \mathrm{CI}=44.0 \%-89.7 \%)$ \\
\hline Specificity & $100.00(95 \% \mathrm{CI}=85.2 \%-100.0 \%)$ \\
\hline Negative likelihood ratio (-LR) & $0.29(95 \% \mathrm{CI}=0.1-0.6)$ \\
\hline
\end{tabular}

Receiver Operating Characteristic (ROC) Curve for identifying osteoporosis by Antegonial index (AI).

Antegonial Index

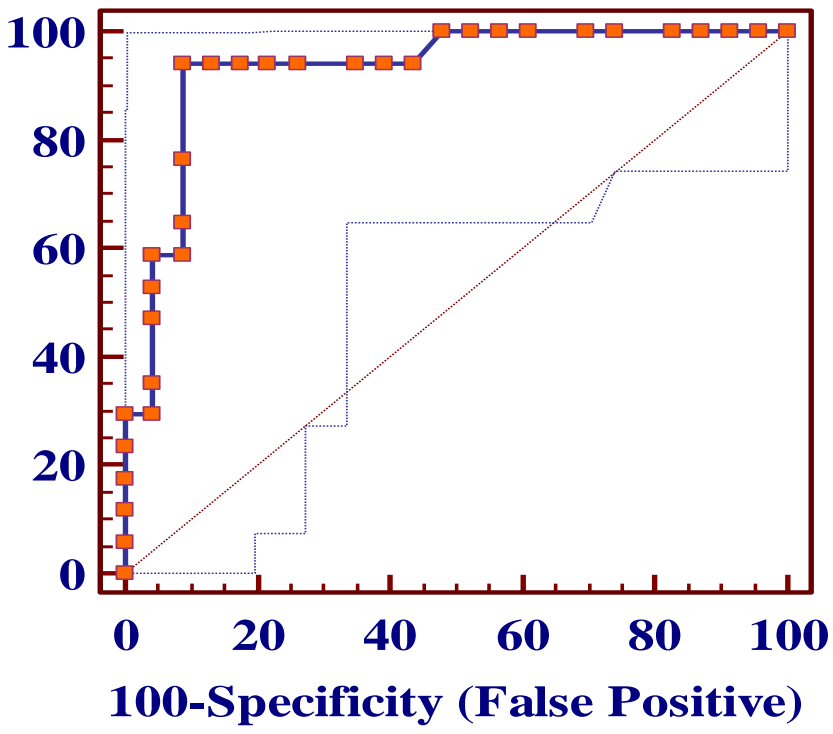


Area under the ROC curve (AUC)

\begin{tabular}{|l|l|}
\hline Area under the ROC curve (AUC) & 0.930 \\
\hline $95 \%$ Confidence interval & 0.802 to 0.986 \\
\hline P value & $0.000(<0.001)$ Significant result \\
\hline
\end{tabular}

Threshold value with highest validity

\begin{tabular}{|l|l|}
\hline Threshold value with Highest validity & $\leq 3.125 \mathrm{~mm}$ \\
\hline Sensitivity & $94.12(95 \% \mathrm{CI}=71.3 \%-99.9 \%)$ \\
\hline Specificity & $91.30(95 \% \mathrm{CI}=72.0 \%-98.9 \%)$ \\
\hline Positive likelihood ratio (+LR) & $10.82(95 \% \mathrm{CI}=2.90-40.90)$ \\
\hline Negative likelihood ratio (-LR) & $0.064(95 \% \mathrm{CI}=0.01-0.40)$ \\
\hline
\end{tabular}

Receiver Operating Characteristic (ROC) Curve for identifying osteoporosis by Mandibular Cortical Index (MCI).

\section{Mandibular Cortical Index}

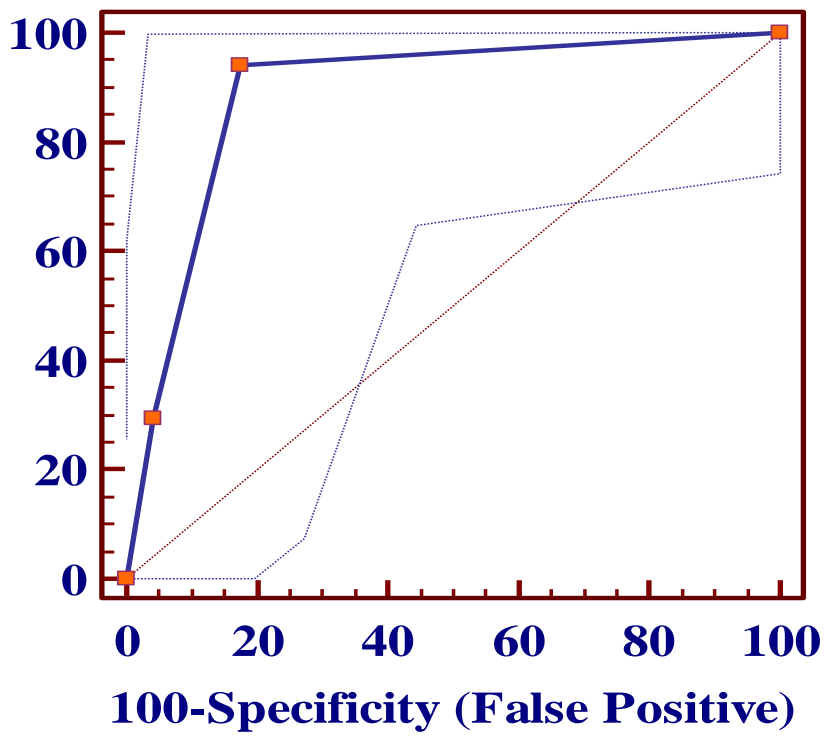

Area under the ROC curve (AUC)

\begin{tabular}{|l|l|}
\hline Area under the ROC curve (AUC) & 0.889 \\
\hline $95 \%$ Confidence interval & 0.749 to 0.966 \\
\hline P value & $0.000(<0.001)$ Significant result \\
\hline
\end{tabular}

Threshold value with highest validity

\begin{tabular}{|l|l|}
\hline Threshold value with Highest validity & $>\mathrm{C} 1$ \\
\hline Sensitivity & $94.12(95 \% \mathrm{CI}=71.3 \%-99.9 \%)$ \\
\hline Specificity & $82.61(95 \% \mathrm{CI}=61.2 \%-95.0 \%)$ \\
\hline Positive likelihood ratio (+LR) & $5.41(95 \% \mathrm{CI}=2.20-13.30)$ \\
\hline Negative likelihood ratio (-LR) & $0.07(95 \% \mathrm{CI}=0.01-0.50)$ \\
\hline
\end{tabular}

Discussion:-

Osteoporosis , a common metabolic disease characterized by reduced bone mass and thinning of cancellous architecture, frequently results in fractures of the vertebrae, hip or forearm. Measurement of bone mineral density is the principal method of diagnosis of osteoporosis because patients with low BMD values have elevated risk of developing a bone fracture. ${ }^{8}$ 
Klemetti E et al (1993) ${ }^{9}$ indicated that particularly the BMD of the buccal part of the mandibular cortex, distally from the foramen mentale, correlates remarkably well with that of the femoral collum which, when BMDs are compared, reflect the general osteoporosis status of the human body. In far resorbed mandibles, the lingual cortex contains significantly less mineral than does the buccal layer and, in that respect, begins to show a correlation with the spongiosa in the same region.

Taguchi A et al (1995) ${ }^{\mathbf{1 0}}$ investigated the possibility of using oral signs as an indicator of postmenopausal osteoporosis. The number of teeth present, mandibular cortical width at the mental region (MCW), the degree of mandibular alveolar bone resorption and morphologic classification of mandibular inferior cortex MIC grade (acc to klemetti E et al ${ }^{5}$ ) were evaluated on panoramic radiographs as oral signs. They concluded that these oral signs revealed a significant relation of age and number of teeth with the fracture status of women from $50-70$ years of age. Calculation of probability of fracture were found to be useful in screening for latent osteoporosis.

Payne JB et (1999) ${ }^{11}$ reported that estrogen deficient postmenopausal women displayed a loss in alveolar bone density using CADIA (Computer assisted densitometric image analysis) Osteoporotic / osteopenic women exhibited a higher frequency of alveolar bone height loss and crestal and subcrestal density loss relative to women with normal BMD. According to the study estrogen deficiency was associated with increased frequency of alveolar bone crestal density loss in osteoporotic/osteopenic women and in the overall study population. Osteoporosis/osteopenia and estrogen deficiency were risk factors for alveolar bone density loss in postmenopausal women.

However, the study conducted by Byung Do Lee et al (2005) ${ }^{\mathbf{8}}$ shows CART (classification and regression tree) analysis which founds that alveolar bone density was less useful than age or strut measures for subjects classification.

Ledgerton D et al (1999) ${ }^{\mathbf{1 2}}$ examined the radiomorphometric indices (GI Gonion index, MI Mental Index, MCI , PMI and AI) and their relationship with age, dentition and social class on panoramic radiographs in British female population and they concluded that BMD differs between ethnic groups so in comparison to white women , black women have a higher peak bone mass so, slower rate of bone loss from femur and spine. All indices demonstrated negative correlation with age and a significant difference between the younger and older age groups. But it was required to test the diagnostic validity of such method relating DXA measurement. They found that dentition has no measurable influence upon MI or PMI.

Iki M et al (2001) ${ }^{\mathbf{1 3}}$ established the criterion value of BMD for the diagnosis of osteoporosis and to estimate the prevalence rate of osteoporosis in Japanese women. They concluded that prevalence rate of osteoporosis at the femoral neck was suggested to be lower in Japanese than in Caucasians, which may account for a lower incidence rate of hip fracture in Japanese women.

Knezovi Zlatari D et al (2001) ${ }^{14}$ conducted that MI, PMI, AI and GI (Gonion Index) showed a general downward trend with age for the both sexes until 75 years of age for female as compared to male and significantly higher incidence of $\mathrm{C} 3$ existed in the oldest group of females.

Devlin $\mathbf{H}$ and Horner K (2002) ${ }^{15}$ demonstrated that the sensitivity and specificity for using panoramic radiographic measurements do not lend themselves to satisfactory diagnosis of osteopenia and osteoporosis. They concluded that these measurements (AI, GI and MI) might be useful as a method of risk assessment when combined with other factors.

Ardakani FE, Niafar N (2004) ${ }^{\mathbf{1 6}}$ identified that it is possible to intercept the progress of osteoporosis through early warning and treatment and they also concluded that the thickness of mandibular angular cortex can be used as a index for bone resorption.

Bozic M, Ihan Hren N (2005) ${ }^{17}$ determined a simple method of common DPT (dental panoramic tomograms) analysis that could represent a simple, inexpensive and readily available screening tool for osteoporosis. Mandibular atrophy was assessed by six anatomic indexes. They concluded RBD ( Relative Bone Density) of the mandible was statistically significantly lower in the osteoporotic group of women.

Lee K et al (2005) $)^{\mathbf{5}}$ were taken 100 women aged 50-84 years who also had BMD assessment of the lumbar spine and femoral neck by dual-energy x-ray absorptiometry. The panoramic images were examined twice by 4 observers 
to evaluate the thickness of the mandibular inferior cortex. They concluded that simple visual estimation of the mandibular inferior cortex on panoramic radiographs may be useful for identifying postmenopausal women with low BMD.

Dargent-Molina P, Piault $\underline{S}$, Bréart G (2005) ${ }^{\mathbf{1 8}}$ on the basis of EPIDOS (Epidemiology of osteoporosis), which was a prospective multicenter study of risk factors for hip fractures concluded that the triage strategy were significantly more sensitive than systematic BMD screening (51 versus 35\%) and would require many fewer BMD examinations .Compared with current recommendations, triage would identify fewer women (20 versus $28 \%$ ) but at a significantly higher average risk of hip fracture. It can be a useful clinical tool for selecting elderly women for treatment or bone densitometry.

White SC et al (2005) ${ }^{\mathbf{1 9}}$ concluded using a Classification and regression tree (CART) that clinical information is as useful as panoramic radiographic information for identifying subjects having low bone mass, and dentists have sufficient clinical and radiographic information to play a useful role in screening for individuals with osteoporosis.

Halling A et al (2005) ${ }^{\mathbf{2 0}}$ concluded that assessment of mandibular cortex patterns is a reliable method to exclude osteopenia/osteoporosis. They indicated that the dentist should screen patients and refer those with positive findings for further assessment for the potential diagnosis of osteopenia/osteoporosis, and also use the strong negative finding (KI category <2) is highly predictive of the absence of osteopenia/osteoporosis as defined by the DXA measurements.

Yüzügüllü B, Gulsahi A, Imirzalioglu P (2009) ${ }^{\mathbf{2 1}}$ concluded that severe erosions on the endosteal margin of the mandible are seen more frequently in women over 60 years in age and mean mandibular cortical width values decrease significantly in women of the same age group.

Kiswanjaya B et al (2010) ${ }^{\mathbf{2 2}}$ concluded that MIC classification may be useful for screening patients for the possibility of osteoporosis by measuring bone stiffness with ultrasound bone densitometry in elderly Japanese people.

Devi Y B K, Nagaraju R, Ravleen N (2011) ${ }^{1}$ concluded that elderly women with PMI $<0.25 \mathrm{~mm}$ in the panoramic radiographs should be referred for advanced osteoporosis investigation. Although radio morphometric indices are regarded as an ancillary method for the diagnosis of osteoporosis on panoramic radiographs, they should be a routine procedure in dental examination and dentists may be able to refer postmenopausal women younger than 65 years for bone densitometry on the basis of incidental findings on dental panoramic radiographs. They stated that the panoramic radiograph plays a vital role in identifying postmenopausal women with undetected osteoporosis.

Peycheva S, Lalabonova H, Daskalov H (2012) ${ }^{23}$ study the role of panoramic radiograph in detecting reliability and accuracy of MCI to diagnose osteopenia and osteoporosis. They concluded that the variations in the width and shape of the inferior cortical edge of the mandible are seen clearly on OPG. Determination of Klemetti index was easy and simple screening method where dentists use an X ray made for other reasons without the patient being additionally burdened radiologically and financially.

Dam A A et al (2013) ${ }^{\mathbf{2 4}}$ concluded that a positive correlation between the CI findings and the presence or absence of osteoporosis as defined by the DVO (German Osteology Organization). Patients were assessed CI of the category "severe" on non-standardised panoramic radiographs have a higher risk of having systemic osteoporosis and suggested that they should be referred to a DXA examination to further verify the diagnosis.

Bozic M, Hren N I (2013) ${ }^{25}$ tested a new method of dental panoramic tomogram (DPT) analysis to show the differences of mandibular bone volume and relative bone density (RBD) in order to detect osteoporosis. DPTs were analyzed using an original method resulting in anatomic and RBD indexes. Average values of RBD indexes were statistically significantly different between $\mathrm{O}$ (Osteoporotic) and N(Normal) and also between o (osteopenic) and $\mathrm{N}$ (Normal) . Important differences regarding RBD were confirmed using a new and original method of common DPT analysis. They concluded that this method could serve as a screening tool for osteoporosis

Sharon H. Chou et al (2014) ${ }^{26}$ indicated that in a multiracial referral population heel BMD correlates significantly with hip BMD and that the ability of heel BMD to predict central osteoporosis as well as presence of vertebral 
fractures is at least as good in AA (African-American) as in Caucasian CA women. Furthermore, heel BMD was more useful than central BMD for identifying patients with glucocorticoid-induced osteoporosis.

\section{Conclusion:-}

1. This is a comparative study for evaluation of Panoramic Mandibular Index (PMI), Antegonial Index (AI), and Mandibular Cortical Index (MCI) of normal and postmenopausal osteoporotic female patients by panoramic radiographs.

2. In conclusion, the present study has proved a positive result in predicting osteoporosis by panoramic radiographs. Hence, panoramic radiographs seem to be a simple, inexpensive tool for screening of osteoporosis among the patients coming for dental evaluation.

3. Our results demonstrate that although radiomorphometric indices are regarded as an ancillary method for the diagnosis of osteoporosis on panoramic radiographs, they should be a routine procedure in dental examination. It is suggested that once these patients are identified then they should be referred to specialist for detailed examination.

\section{References:-}

1. B.K. Yashoda Devi 1, Nagaraju Rakesh 2, Nagi RavleenDiagnostic efficacy of panoramic mandibular index to identify postmenopausal women with low bone mineral densities. J Clin Exp Dent. 2011;3(5):e456-61.

2. Leite AF, Figueiredo PT, Guia CM, Melo NS, Paula APD. Correlations between seven panoramic radiomorphometric indices and bone mineral density in postmenopausal women. Oral Surg Oral Ned Oral Pathl Oral Radiol Endod. 2010; 109:449-456.

3. A Gulsahi, B Yu"zu"gu" llu", P I'mirzalıg־lu and Y Genc, Dentomaxillofacial Radiology (2008) 37, 288-292 RESEARCH Assessment of panoramic radiomorphometric indices in Turkish patients of different age groups, gender and dental status

4. Celia Regina Winck Mahl, Renata Licks, Vania Regina Camargo Fontanella Comparison of morphometric indices obtained from dental panoramic radiography for identifying individuals with osteoporosis/osteopenia Radiol Bras. 2008 Mai/Jun;41(3):183-187

5. Lee K, Taguchi A, Ishii K, Suei Y, Fujita M, Nakamoto T, et al. Visual assessment of the mandibular cortex on panoramic radiographs to identify postmenopausal women with low bone mineral densities. Oral Surg Oral Med Oral Pathol Oral RadiolEndod 2005; 100: 226-231

6. D Ledgerton1, K Horner*, H Devlinand H Worthington Radiomorphometric indices of the mandible in a British female population Dentomaxillofacial Radiology (1999) 28,173: 18.

7. GauR. B Evaluation of panoramic Radiographs as a Screening Tool of Osteoporosis in Post Menopausal Women: A Cross Sectional Study Journal of Clinical and Diagnostic Research. 2013 Sept, Vol-7(9): 20512055

8. Byung Do Lee, Stuart C.White,Iksan Age and trabecular features of alveolar bone associated with osteoporosis OOOE 2005;100:92-8.

9. Scand J Dent Res 1993 Cortical bone mineral density in the mandible and osteoporosis status in postmenopausal women:101:219-23

10. A. Taguchi, keiji Tanimoto, Yoshikazu Suei, Keiko Otani , and Takuro wada Oral signs as indicators of possible osteoporosis in elderly women OOOE 1995;80:612-6.

11. PayneJB, Reinhardt RA, Nummikoski PV, Patil KD. Longitudinal alveolar bone loss in postmenopausal osteoporotic / osteopenic women.Osteoporos Int 1999;10:34-40.

12. D Ledgerton1, K Horner*, H Devlinand H Worthington Radiomorphometric indices of the mandible in a British female population Dentomaxillofacial Radiology (1999) 28,173: 18

13. M. Iki et al Bone Mineral Density of the Spine, Hip and Distal Forearm in Representative Samples of the Japanese Female Population: Japanese Population-Based Osteoporosis (JPOS) Study Osteoporos Int (2001) 12:529-537.

14. D. Knezovi Zlatari et al Influence of Age and Gender on Radiomorphometric Indicesof the Mandible in Removable Denture Wearers Radiomorphometric Indices, Coll. Antropol.25(2001) 2: 259-266.

15. H. Devlin and K. Horner Mandibular Radiomorphometric Indices in the Diagnosis of Reduced Skeletal Bone Mineral Density Osteoporos Int (2002) 13:373-378.

16. Fatemeh Ezoddini and Nasim Niafar Evaluation of changes in a mandibular angular cortex using panoramic images The Journal of contemporary dental practice vol 5 no 3 aug 15, 2004 
17. M Bozic and N Ihan Hren RESEARCH Osteoporosis and mandibles Dentomaxillofacial Radiology (2005) $35,178-184$.

18. P. Dargent-Molina S. Piault G. Breart A triage strategy based on clinical risk factors for selecting elderly women for treatment or bone densitometry: the EPIDOS prospective study Osteoporos Int (2005) 16: 898-906

19. Stuart C. White et al Clinical and panoramic predictors of femur bone mineral density Osteoporos Int (2005) 16: 339-346.

20. Halling. A et al Comparison between the Klemetti index and heel DXA BMD measurements in the diagnosis of reduced skeletal bone mineral density in the elderly Osteoporos Int (2005) 16: 999-1003.

21. Yüzügüllü et al Radiomorphometric indices and their relation to alveolar bone loss in completely edentulous Turkish patients: A retrospective study J Prosthet Dent 2009;101:160-165

22. B. Kiswanjaya et al Relationship between the mandibular inferior cortex and bone stiffness in elderly Japanese people Osteoporos Int (2010) 21:433-438

23. Stefka Peycheva, Hristina Lalabonova, Hristo Daskalov Early detection of osteoporosis in patients over 55 using orthopantomography Journal of IMAB2012.

24. Al-Dam .A et al Mandibular cortical shape index in non-standardised panoramic radiographs for identifying patients with osteoporosis as defined by the German Osteology Organization Journal of Cranio-Maxillo-Facial Surgery 41 (2013) 165- 169.

25. Marko Bozic, Natasa Ihan Hren A novel method of dental panoramic tomogram analysis: A perspective tool for a screening test for osteoporosis Journal of Cranio-Maxillo-Facial Surgery 41 (2013) 808e815

26. Sharon H. Chou et al Utility of Heel Dual-Energy X-ray Absorptiometry in Diagnosing Osteoporosis Journal of Clinical Densitometry: Assessment \& Management of Musculoskeletal Health,vol. 17, no. 1, 16e24, 2014 by The International Society for Clinical Densitometry 1094-6950/17:16 